\title{
IdeAs
}

Idées d'Amériques

\section{Politique urbaine dans l'Amérique de Donald Trump : tournant néolibéral, impact des coupes budgétaires et plan de rénovation des infrastructures}

\section{Laurence Gervais}

\section{OpenEdition}

\section{Journals}

Édition électronique

URL : https://journals.openedition.org/ideas/4427

DOI : $10.4000 /$ ideas.4427

ISSN : 1950-5701

Éditeur

Institut des Amériques

\section{Référence électronique}

Laurence Gervais, «Politique urbaine dans l'Amérique de Donald Trump : tournant néolibéral, impact des coupes budgétaires et plan de rénovation des infrastructures », IdeAs [En ligne], 12 | 2018, mis en ligne le 14 novembre 2018, consulté le 20 octobre 2022. URL : http://journals.openedition.org/ideas/ 4427 ; DOI : https://doi.org/10.4000/ideas.4427

Ce document a été généré automatiquement le 20 octobre 2022

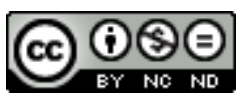

Creative Commons - Attribution - Pas d'Utilisation Commerciale - Pas de Modification 4.0 International - CC BY-NC-ND 4.0

https://creativecommons.org/licenses/by-nc-nd/4.0/ 


\title{
Politique urbaine dans l'Amérique de Donald Trump : tournant néolibéral, impact des coupes budgétaires et plan de rénovation des infrastructures
}

\author{
Laurence Gervais
}

1 Les propositions du président D. Trump - dès sa campagne - en termes de politique urbaine, ont constitué un tournant historique dans la façon dont le gouvernement fédéral envisage les problèmes urbains et leurs solutions, une approche de « rénovation urbaine » fondée sur une gouvernance néolibérale ayant recours aux partenariats avec le secteur privé, aux crédits d'impôts et à un financement local accru, mais dépourvue de politique en faveur du logement et menant une lutte contre les villes-sanctuaires et l'immigration illégale.

2 Lorsque B. Obama a été élu en 2008, il a défrayé la chronique en se présentant comme le président des villes, voire des centres-villes. Son administration a créé des agences gouvernementales pour le développement durable, et a lancé des partenariats et des initiatives en coopération avec les pouvoirs locaux.

3 Pourtant, l'urgence de régler les problèmes liés à la crise économique a été plus forte que celle de s'attaquer aux problèmes urbains en profondeur. L'une des grandes déceptions pour les urbanistes reste que le gouvernement Obama n'a pas passé de nouvelle loi sur les transports. La politique de grands travaux qui devait attribuer 17 milliards de dollars aux villes pour la reconstruction de nouveaux logements (American Jobs Act) n'a pas non plus été passée. Le programme HAMP (Home Affordable Modification Program), a de son côté été moins financé que prévu. C'est notamment en s'appuyant sur ces échecs de la politique de la ville du gouvernement Obama, que le candidat Trump a défini ses objectifs de campagne, en insistant sur le fait que la situation des Afro-Américains et des habitants des centres-villes n'avait pas été améliorée. 
4 En 2014 la ligue nationale des villes (National League of Cities, NLC ${ }^{1}$ ) identifiait dix problèmes clé à traiter en priorité dans les villes étasuniennes, dont l'infrastructure (transports, routes, adduction de l'eau), l'accès à l'éducation, le logement, la sécurité, l'immigration et l'accès à la citoyenneté et l'environnement. Cet article se propose de revenir d'une part sur les propositions de Trump durant sa campagne en regard de ces problèmes majeurs identifiés par les villes elles-mêmes et d'autre part sur les effets du budget fédéral et des coupes budgétaires sur ces mêmes problèmes urbains.

\section{Les objectifs de politique urbaine de 2016 de Trump : «Une Rénovation urbaine pour la sécurité, l'emploi, l'éducation et la rénovation des infrastructures »}

5 Le 26 octobre 2016, à Charlotte, Caroline du Nord, dans un discours de campagne, le candidat Trump a promis l'avènement d'un plan de "rénovation urbaine» qui assurerait la sécurité des villes aux États-Unis, créerait des emplois, améliorerait l'accès à l'éducation et rénoverait les infrastructures, notamment dans les quartiers habités par les Afro-Américains (Mozee S. \& Davis M., $2017: 2$ ). Le candidat a plusieurs fois fait référence aux villes étasuniennes comme à des « zones de guerre » et a déclaré que les conditions de vie y étaient « inacceptables » (Mozee S. \& Davis M., 2017 : 4).

\section{Les Propositions de Trump face aux problèmes majeurs listés par la NLC}

6 Les grandes lignes des propositions de Trump visant à régler les problèmes les plus saillants listés par la NLC en 2016 étaient les suivantes :

7 En matière d'éducation, l'objectif était de maintenir une politique de "choix" permettant aux enfants issus de milieux défavorisés d'aller dans n'importe quelle école qu'elle soit privée, publique, semi-privée sous contrat (charter schools), religieuse ou non, ou même d'opter pour l'instruction à domicile. Le candidat Trump avait aussi l'intention de mettre en place une commission d'enquête sur le lien entre école et prison, de financer les universités et les collèges afro-américains historiques et de favoriser les cycles courts.

8 En ce qui concerne la sécurité, Trump affichait l'intention de financer et investir dans le renforcement de l'application de la loi afin de combattre les gangs, le trafic de drogue et les cartels criminels dans les quartiers.

9 Sur le plan fiscal, Trump prévoyait de mettre en place des politiques d'avantages fiscaux pour attirer les entreprises étrangères dans les quartiers en difficulté et de donner la possibilité aux villes et aux Etats de demander le classement de zones défavorisées en zones sinistrées afin d'initier la reconstruction de leurs infrastructures, de permettre la démolition des immeubles abandonnés et de renforcer l'application de la loi.

10 En termes d'emploi, Trump entendait mettre en place des réformes financières afin de faciliter l'accès au crédit des jeunes Afro-Américains pour créer des entreprises, d'encourager la création de moyennes et petites entreprises en permettant aux 
travailleurs sociaux de transformer les allocations sociales en micro-crédit. Il s'agit donc en clair de limiter les aides publiques et de privilégier l'emprunt privé.

11 Un des points forts de sa campagne était la protection contre l'immigration illégale. Il entendait donc réformer les règles concernant les visas afin de donner la préférence aux travailleurs américains et de suspendre l'admission de réfugiés dans les villessanctuaires afin de réinvestir l'argent ainsi économisé dans les centres-villes.

Enfin, en termes d'infrastructure Trump voulait mettre en place des partenariats public-privés et favoriser l'investissement privé grâce à des crédits d'impôts et des politiques fiscales en faveur de l'investissement, censés dégager mille milliards de dollars sur les dix années à venir dans les centres-villes. Il entendait également annuler toutes les politiques environnementales en faveur du climat mises en place sous les administrations Clinton et Obama y compris les versements aux Nations unies pour lutter contre le réchauffement climatique, ce qui devait permettre de dégager 100 milliards de dollars en huit ans afin de reconstruire les infrastructures, y compris l'adduction de l'eau, dans les centres villes américains.

On notera que parmi ces propositions, si certaines s'adressaient aux problèmes listés par la NLC, rien n'était prévu en faveur du logement, et que les mesures en direction du climat étaient des mesures négatives. Par ailleurs, les mesures en faveur de la sécurité étaient des mesures de renforcement de l'application de la loi, à l'opposé des positions d'Obama. Enfin, les relations difficiles de Trump avec un certain nombre de maires de grandes villes marqués à gauche risquaient de générer des situations conflictuelles, notamment à propos de l'immigration. Beaucoup d'entre eux étant en faveur d'une politique d'ouverture des frontières et à la tête de "villes-sanctuaires" où la municipalité refuse d'appliquer les lois fédérales sur l'immigration. Beaucoup de villes en décroissance démographique telles que Detroit ou Dayton considèrent les immigrants comme leur seul espoir de repopulation. Or Trump a promis de couper les vivres aux villes-sanctuaires, et beaucoup de maires de ces villes sont bien décidés à le défier.

\section{Le Budget fédéral, les coupes budgétaires ayant un effet sur les villes}

14 En mars 2016, les recommandations du think tank conservateur The Heritage Foundation $^{2}$ en matière de budget allaient vers une réduction des dépenses de 10 mille milliards de dollars entre 2017 et 2029. La plupart des propositions de Heritage ont été utilisées par les membres du gouvernement Trump pour définir les coupes budgétaires proposées en 2018 dans les ministères et les programmes dédiés aux villes.

\section{Logement}

15 Heritage proposait une réduction des fonds alloués au ministère du Logement (HUD) de 4,3 milliards de dollars pour la seule année 2017 et de 292,8 milliards de dollars entre 2017 et 2026. Une des propositions était de transférer la responsabilité fiscale des programmes de logements sociaux publics aux Etats. Une autre proposition était de supprimer la dotation globale pour le développement local (Community Development Block Grant) qui gère l'amélioration et la création de logements publics, les services 
publics et les offices de logements publics locaux et les prêts subventionnés pour l'accès à la propriété. A terme, la FHA (Federal Housing Administration) qui gère le logement public au niveau national devait disparaître.

\section{Environnement et énergie}

Heritage proposait également de faire une économie de 10 milliards de dollars sur les dépenses du ministère de l'énergie, en vendant au secteur privé les actifs publics tels que les réserves de pétrole. Par ailleurs un large panel de propositions étaient faites pour démanteler les programmes de réduction d'émissions de carbone et de règlementation de la pollution et pour réduire les fonds alloués aux programmes d'efficacité energétiques du ministère de l'Energie, tels que ceux dédiés à la construction d'immeubles à énergie positive. Ceci viendrait s'ajouter à la suppression des subventions aux énergies propres et de neuf programmes climatiques. Les règlementations en matière de gaz à effet de serre ainsi que la loi sur la protection de l'eau devraient également être supprimées.

\section{Transports}

17 En ce qui concerne les transports, Heritage proposait une diminution de 21,9 milliards de dollars des fonds alloués au ministère des Transports pour la seule année 2017 et de 216,6 milliards entre 2017 et 2026. Le budget prévoyait également la diminution des allocations aux programmes de transports publics des Etats et la fin du financement du transport public interurbain AMTRACK et WMATA. Un certain nombre de programmes de transports publics locaux ne seraient plus financés, impliquant que des offres seraient lancées en direction d'entreprises privées pour les remplacer, ou du moins que des appels seraient fait en vue de collecter des fonds privés et de créer des partenariats publics-privés pour compenser la perte en lignes de transports urbains et interurbains.

\section{Justice et sécurité}

18 Une réduction du budget de la justice de 3,77 milliards de dollars était envisagée par Heritage pour la seule année 2017, grâce à la suppression de financements tels que celui $\mathrm{du}$ Office of Community Oriented Policing Services, des subventions aux organismes de lutte contre la violence faite aux femmes, à l'aide juridique, aux associations de défense des droits civiques et de l'environnement.

\section{Éducation}

Enfin, en termes d'éducation, les coupes budgétaires recommandées étaient de 16,8 milliards de dollars pour 2017 et 236,06 milliards de dollars pour la période s'étalant de 2017 à 2026. Le rapport proposait d'éliminer les dotations globales du ministère de l'Education et de les remplacer par un système d'allocations aux zones d'éducation défavorisées. Au niveau de l'enseignement supérieur, le rapport préconisait de réduire les aides sous forme de bourses ou de prêts subventionnés aux étudiants afin de favoriser l'emprunt privé. 


\section{Crise de l'infrastructure dans les villes : Le Plan pour l'infrastructure de février 2018}

En février 2018, le « Plan pour l'infrastructure » du président Trump a été rendu public. L'un des points saillants du plan était que les villes étaient invitées à utiliser leurs propres fonds pour en réaliser les objectifs.

\section{Infrastructure dans les transports}

21 En dépit des critiques du président Trump à propos de l'infrastructure et notamment de la vétusté des transports publics lors de sa campagne présidentielle, le «Plan pour l'infrastructure " suit les recommandations de Heritage. Il propose une dépense d'à peine 200 milliards de dollars sur 10 ans, soit à peine $20 \%$ de plus que ce que le pays dépense déjà grâce à une loi passée sous l'administration Obama.

\section{Infrastructure dans le logement}

La proposition de budget pour l'année fiscale 2019, publiée en février 2018 sous la forme du Bipartisan Budget Act de 2018 et du Continuing Appropriations Act de 2018, a confirmé l'intention de mettre en place une politique d'austérité dans les villes. Beaucoup des réductions budgétaires liées au logement devraient affecter les programmes d'aide aux familles défavorisées. Dans le projet de budget, il est prévu une coupe de 8,8 milliards de dollars au HUD, soit une diminution de 18,3\% par rapport aux dépenses actuelles. Comme préconisé par Heritage en 2017, le budget actuel propose la suppression d'un certain nombre de dotations globales dont le "Community Development Block Grant » allouées aux gouvernements locaux pour le développement et l'entretien de leurs infrastructures. Le Home Investment Partnerships Program, une dotation globale destinée à aider les municipalités dans leurs efforts de construction de logements abordables, jugée "dépassée ", sera entièrement supprimée, ainsi que le Public Housing Capital Fund, destiné à la réhabilitation du logement public. Parmi les autres programmes supprimés, on note le Self-Help Homeownership Opportunity Program pour l'aide à l'accès à la propriété, et le Choice Neighborhood Initiative, qui finançait des programmes de réhabilitation des quartiers défavorisés grâce à des fonds privés et publics. On note également une diminution de $11,2 \%$ des aides au logement pour les locataires sous forme d'allocations et de programmes de logements publics. Enfin le budget réitère l'intention du gouvernement à terme de conditionner l'obtention de ces aides à des exigences en matière de retour au travail.

\section{Conclusion}

Il semble donc clair d'une part que le budget Trump pour 2019 va suivre les recommandations ultra-conservatrices de la Fondation Heritage notamment en termes d'économies sur les transports publics et sur le logement public. Bien que le candidat Trump ait critiqué les échecs de la politique de la ville du gouvernement Obama en matière de logements et l'état délabré de certaines villes, il semble que son Plan pour l'Infrastructure limite davantage encore les aides de l'Etat fédéral tout en préconisant des plans de rénovation urbaine au niveau local. Ceci indique clairement une intention 
de demander localement aux Etats et aux villes d'avoir recours à des fonds privés et à des partenariats publics-privés, comme c'est déjà le cas dans un grand nombre de villes qui ont mis en place une gouvernance néolibérale et n'envisagent plus de construction de logement purement public ${ }^{3}$. En ce qui concerne l'environnement et l'énergie, la position du gouvernement Trump est claire et suivra sans doute les recommandations de Heritage. Il n'est pas encore avéré que les économies préconisées par Heritage donneront lieu à des coupes budgétaires dans l'éducation, toutefois la décision de maintenir une politique de " choix » dans l'éducation devrait encourager la création de « charter schools » et d'écoles privées au niveau local et la fermeture d'écoles publiques dans les quartiers défavorisés comme cela a déjà été le cas dans de nombreuses villes depuis vingt ans. Enfin, la suppression d'un grand nombre de dotations globales dans le logement, le transport, l'éducation et l'environnement va dans le sens de l'utilisation de crédit d'impôts.

L'approche de « rénovation urbaine " préconisée par le candidat Trump risque d'aller dans le sens du déplacement des populations pauvres qui n'auront plus accès à des services publics (logement, transports). Les politiques de revitalisation des espaces publics en centre ville devront se faire sur les deniers locaux, incluant la privatisation des services, la dérèglementation et le recours aux fonds privés pour assurer ces services pour équilibrer les budgets des villes.

\section{BIBLIOGRAPHIE}

Ratcliffe, Michael, A Century of Delineating a Changing Landscape: The Census Bureau's Urban and Rural Classification, 1910 to 2010, Washington, DC ., Geography Division: US Census Bureau, 2015.

Mozee, Sam \& Melvin Davis, «A Preliminary Look at president Trump's Proposed urban Agenda », Research Brief, Mississipi Urban Research Center, College of Public Service, Jackson State University, vol. 2, n 4, March 2017, p. 1-13.

\section{Ressources électroniques}

https://www.heritage.org/budget-and-spending/report/blueprint-reform-comprehensivepolicy-agenda-new-administration-2017.

https://www.congress.gov/bill/115th-congress/house-bill/1892/text?

$q=\% 7 B$ \%22search \%22 \%3A \%5B \%22bipartisan+budget+act+2018 \%22 \%5D \%7D\&r =1

https://www.congress.gov/bill/115th-congress/house-bill/601/text

https://www.whitehouse.gov/wp-content/uploads/2018/02/budget-fy2019.pdf

https://www.congress.gov/bill/115th-congress/house-bill/1892/text ?

$q=$ \%7B \%22search \%22 \%3A \%5B \%22bipartisan+budget+act+2018 \%22 \%5D \%7D\&r =1

https://www.congress.gov/bill/115th-congress/house-bill/601/text 


\section{NOTES}

1. La NLC est une organisation à but non lucratif qui représente les intérêts de 49 associations municipales représentant elles-mêmes 19000 villes et villages.

2. En mars 2016 et en janvier 2017 Heritage a publié deux rapports, Blueprint for Reform et Blueprint for Balance, recommandant des coupes budgétaires drastiques.

3. Comme à Chicago par exemple, depuis l'administration Daley, avec l'utilisation de partenariats publics-privés afin de construire des logements «mixtes ». Dans le cadre de ces partenariats, l'argent des taxes foncières revient directement aux promoteurs privés prenant part à la réhabilitation et non à la ville. Cet argent peut aussi être utilisé pour attirer de nouveaux commerces à qui l'on promet des avantages fiscaux (crédits d'impôts), ou encore revenir à des programmes d'investissement dans les commerces locaux.

\section{AUTEUR}

\section{LAURENCE GERVAIS}

Professeure des universités - Université Paris Nanterre. logl@free.fr 